\title{
MELANOMA DE PENE
}

\author{
M.L. MILLÁN SAGASTE, L.A. ASENSIO LAHOZ*, J.M. MONGE MIRALLAS*, \\ A. PÉREZ MARTÍN, M.J. FERNÁNDEZ LERONES, A.J. GUEZMES DOMINGO**, \\ O. ACINAS GARCÍA**
}

*Servicio de Urología. **Servicio de Anatomía Patológica. Centro de Salud La Vega-Zapatón. Torrelavega. Cantabria.

Actas Urol Esp. 27 (2): 152-154, 2003

\section{RESUMEN}

"MELANOMA DE PENE"

Presentamos un caso de melanoma maligno de pene. Su rareza inferior al 1\% de las neoplasias malignas de genitales masculinos, la ausencia de protocolos estandarizados como para otras neoplasias, nos impulsan su publicación.

PALABRAS CLAVE: Melanoma maligno. Pene.

\section{ABSTRACT}

"MELANOMA OF THE PENIS"

We present a case of penis' malignant melanoma. The inferior rarity to 1\% of masculine genital's malignant neoplasms, the absense of standardised protocols as for other neoplasm's, impel us to its publication.

KEY WORDS: Malignant melanoma. Penis.

$\mathrm{E}$ 1 melanoma primario de pene es una localización infrecuente, sólo supone el 1\% de las neoplasias malignas de genitales masculinos. Es más frecuente en glande que en uretra, si bien también puede afectar a meato, prepucio o surco coronal.

El melanoma de pene metastatiza precozmente a ganglios linfáticos (el 40\% en el momento del diagnóstico, según la mayoría de las series), también a distancia (hígado, pulmón, hueso y cerebro).

Al igual que en otras localizaciones el tratamiento incluye cirugía agresiva y opcionalmente radioterapia, quimioterapia e inmunoterapia complementarias.

En cuanto al pronóstico viene determinado por el estadio y por la invasión tumoral. A los cinco años la supervivencia es inversamente proporcio- nal a la profundidad invasiva del tumor. En general tiene peor pronóstico si afecta a mucosas ya que el descubrimiento suele ser más tardío ${ }^{1}$.

\section{CASO CLÍNICO}

Paciente de 61 años de edad con antecedentes personales de hipertensión arterial (sin tratamiento médico). Sin otros antecedentes de interés ni tratamiento habitual. Consulta enviado por Atención Primaria por lesión papular, hiperpigmentada, de tamaño similar a "una lenteja" localizada en glande que tiene desde hace aproximadamente año y medio, y que recientemente ha crecido junto con aparición de otra más pequeña en surco balanoprepucial. Ninguna otra sintomatología acompañante. Respecto a la exploración física las lesiones son de bordes irregulares, sin ulceración y de coloración negruzca (Fig. 1). No se palpan adenopatías. 


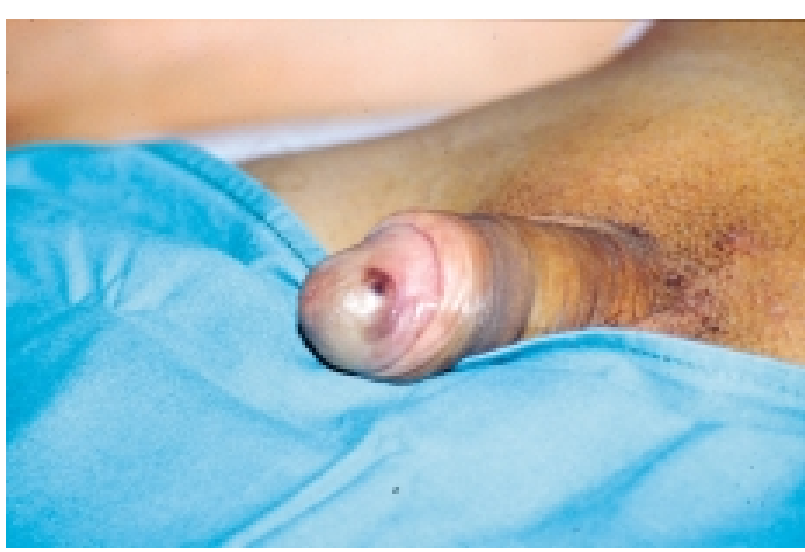

FIGURA 1. Aspecto que presentaba la lesión a la exploración fisica.

Tras una analítica rutinaria que es absolutamente normal se decide la realización de extirpaciónbiopsia, siendo el resultado anatomopatológico de: melanoma nodular maligno de pene, estadio Clark III-IV, infiltra dermis reticular superficial, Breslow $4,2 \mathrm{~mm}$ y que respeta bordes quirúrgicos pT4b (tumor de $>4 \mathrm{~mm}$ grosor) y satelosis a dos centímetros del tumor primario (Fig. 2).

Se realizó estudio de extensión TAC torácico y abdominopélvico, y Rx tórax, siendo negativos para metástasis.

Tras sesión clínica junto con Oncología y las pocas series y protocolos en marcha se decidió la realización de penectomía parcial con linfadenectomía ilio-inguinal superficial bilateral. La anatomía patológica confirmó la ausencia de tumor residual y de ganglios metastásicos. Se detectaron

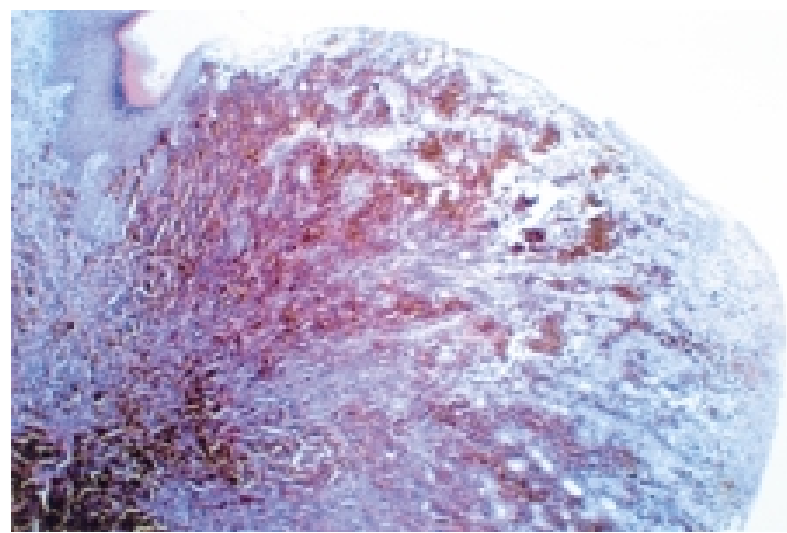

FIGURA 2. Foto microscópica (anatomia patológica). Melanoma maligno nodular: Ulcera superficialmente el epitelio escamoso. Las células presentan abundante pigmento melánico intracitoplasmático (He $x$ 4). pequeñas lesiones en glande etiquetadas como lentiginosis genital (Fig. 3). Resto sin alteraciones. Tras los resultados de la pieza se decidió en nueva sesión no administrar ningún otro tipo de tratamiento. El paciente no presentó ninguna complicación salvo ligero edema en extremidad inferior izquierda. A los seis meses y al año las revisiones $\mathrm{y}$ estudios complementarios son negativos de extensión de la enfermedad.

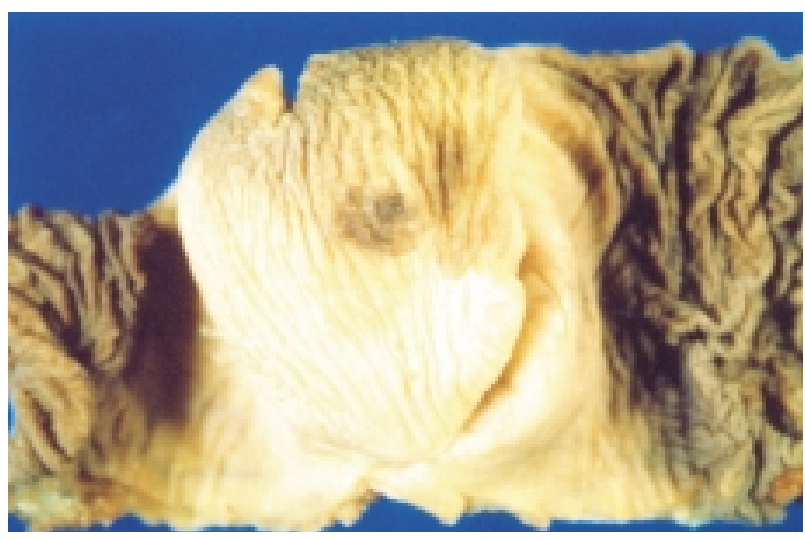

FIGURA 3. Foto macroscopica (anatomia patológica). Segmento anterior de pene: Estado postresección de glande por melanoma maligno nodular. Lentiginosis genital.

\section{DISCUSIÓN}

Revisada la bibliografía sobre el melanoma maligno de pene, apenas existen unos 140 casos descritos. Es un tumor que puede presentarse a cualquier edad, si bien parece más frecuente en el grupo de población con edades entre 60 y 80 años. La forma de presentación y los síntomas son variables, generalmente son lesiones pre-existentes durante meses o años que recientemente han sufrido crecimiento o ulceración. En otros casos los pacientes refieren aparición reciente de una lesión pequeña, marrón/negruzca, papular o pediculada $^{1,2}$.

Todos los artículos publicados coinciden en el retraso en el diagnóstico y por consiguiente en el mal pronóstico de la enfermedad, apuntando como causas, la inespecificidad de los síntomas, el pudor, falta de reconocimiento por el clínico, etc.

El pronóstico se ensombrece por la localización anatómica que impide una cirugía con márgenes generosos con el consiguiente aumento de la recurrencia y la rica vascularización y drenaje linfático que determina una temprana diseminación $n^{3,4}$. 
La actitud terapéutica es un tema aún por resolver, en tanto que no existen criterios claros ni unificados dada la baja incidencia de la enfermedad. El tratamiento esencial debe ser siempre quirúrgico, realizando penectomía parcial en estadio I de Bracken-Diokno (lesión confinada a pene) discutible si linfadenectomía bilateral o linfadenectomía del ganglio centinela. Nos inclinamos por la linfadenectomía bilateral, sin ningún otro tipo de tratamiento complementario. Los estadios II con ganglios linfáticos positivos y III con metástasis a distancia precisaran de otros tipos de tratamiento complementario radio, quimio o inmunoterapia según protocolos ${ }^{5-7}$.

\section{CONCLUSIÓN}

Ante toda lesión "sospechosa" hiperpigmentada debe plantearse la extirpación completa con márgenes libres ante la sospecha de melanoma, ya que el diagnóstico temprano es el único factor que mejora la supervivencia de esta enfermedad.

\section{REFERENCIAS}

1. ZAMORA PARRA B, CASADO PÉREZ C.: I Ponencia oficial de melanoma. Serv. Cirugía Plástica. H. General Yagüe. Ed. ENE Ediciones 1998: 86.

2. PARRA MUNTANER L, MADRID GARCÍA FJ, SÁNCHEZ MERINO JM, SÁNCHEZ SÁNCHEZ E, GONZÁLEZ GONZÁLEZ O, GÓMEZ CISNEROS SC, LÓPEZ PALACIOS JC, GARCÍA ALONSO J.: Melanoma primario de pene: a propósito de un caso. Arch Esp Urol 2000 may; 53 (4): 375-376.

3. DE BREE E, SANIDAS E, TZARDI M, GAKI B, TSIFTSIS D.: Malignant melanoma of the penis. Eur $J$ Surg Oncol 1997 jun; 23 (3): 277-279.

4. LARSSON KB, SHAW HM, THOMPSON JF, HARMAN RC, McCARTHY WH.: Primary mucosal and glans penis melanomas: the Sydney Melanoma Unit experience. Aust NZ Surg 1999 feb; 69 (2): 121-126.

5. CHABANNES E, WALLERAND H, BERNARDINI S, DEBIERE F, ALLOUC H, BITTARD H.: Malignant penile melanoma. Prog Urol 2000 feb; 10 (1): 101-105.

6. ZURRIDA S, BARTOLI C, CLEMENTE C, DE PALO G.: Malignant melanoma of the penis. A report of four cases. Tumori 1990 dec 31; 76 (6): 599-602.

7. STILL WELL TJ, ZINCKE H, GAFFEY TA, WOODS JE.: Malignant melanoma of the penis. $J$ Urol 1998 jul; 140 (1): $72-75$.

Dr. L.A. Asensio Lahoz

C/ Consuelo Bergés, 16 - portal 2, 2ํㅗ

39012 Santander (Cantabria)

(Trabajo recibido el 25 abril de 2002) 